\title{
LIQUID CRYSTAL NONLINEAR OPTICS
}

\author{
I.C. Ḱно \\ The Pennsylvania State University, Department of Electrical and Computer Engineering \\ University Park, PA 16802, USA
}

\begin{abstract}
A review of liquid crystal nonlinearities and nonlinear optical phenomena observed in all phases of liquid crystals is presented. Recent observation of self-starting optical phase conjugation in nematic liquid crystal is specifically discussed as an example of a novel nonlinear optical phenomenon.
\end{abstract}

PACS numbers: 42.65.-k, 42.65.IIw, 42.65. Vh, 61.30.-v

\section{Introduction}

Liquid crystals occupy a very important and unique place in current research and development efforts in various display and electro-optical devices. Research conducted in the last decade has also shown that they are optically highly nonlinear materials, and can be applied to practical devices and systems. Among the many noteworthy features of liquid crystals are the following [1, 2]:

- $\Lambda$ large birefringence and good transparency spanning the near UV to far infrared spectrum (cf. Fig. 1).

- Extraordinary large optical nonlinearities of various forms suitable for applications with $\mathrm{cw}$-picosecond laser pulses.

- Almost inexhaustible possibilities for synthesizing new compounds or mixtures of liquid crystals, or liquid crystals and other organic molecular systems, for optimizing material nonlinear optical responses or device performance.

- Fluid-like and crystalline properties that allow the fabrication of thin film devices or optical guided wave structures (fibers or planar waveguide) with great flexibility in configurations. Indeed, out recent studies have conclusively demonstrated the feasibility of fabricating liquid crystalline optical fibers of various dimensions, and the generation of several nonlinear optical effects with high-efficiency/low-threshold.

Other phases of liquid crystal also possess distinctive properties well suited for some specialized applications. In smectics, the lower degree of scattering loss and the presence of ferroelectricity allows longer interaction length (up to around $0.5 \mathrm{~mm}$ ) and symmetry-breaking type of harmonic generation processes. In the isotropic phase, there is an advantage of much longer interaction length (up to 

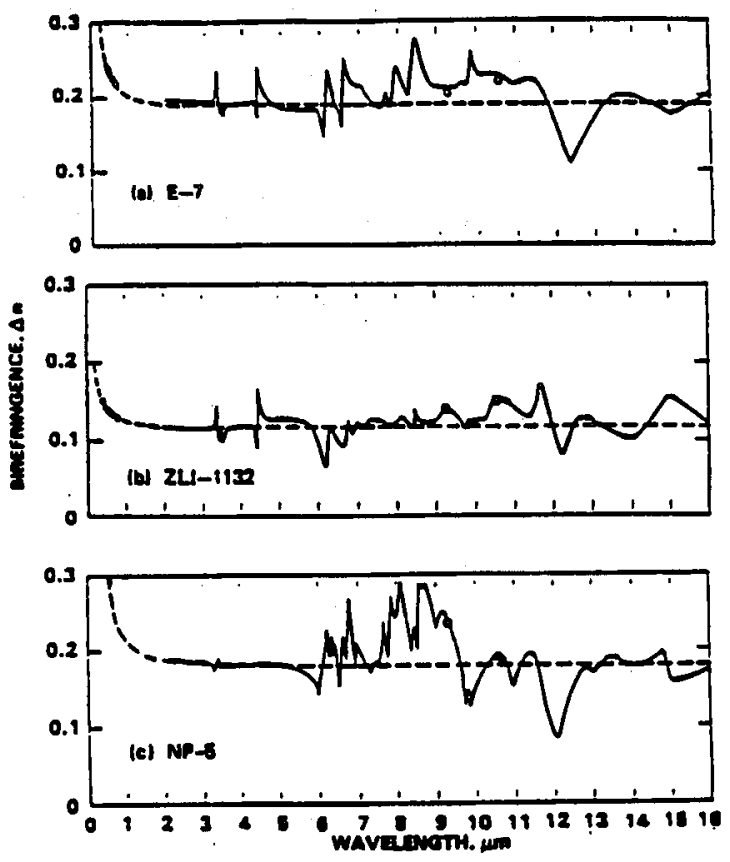

Fig. 1. Birefringence of the liquid crystal E7, ZL1-1132 and NP-5 over the visible-infrared spectral regime.

centimeters compared to microns for nematics) and sizeable nonlinearities [1-3]. In addition to all these special characteristics pertaining to specific mesophases, there is an overall advantage enjoyed by liquid crystals, namely, a well-established synthesis science which fuels the current rapid emergence of numerous new advanced liquid crystal materials.

\section{Optical nonlinearities of liquid crystals}

Optical properties of pure liquid crystals can be described by two distinctively different kinds of model depending on whether the fundamental mechanisms are electronic or non-electronic. The electronic nonlinearities of liquid crystals, such as $\chi^{(2)}(-2 \omega ; \omega, \omega, \omega)$ and $\chi^{(3)}(-3 \omega ; \omega, \omega, \omega)$ for second and third harmonic generations, respectively, require a quantum mechanical approach where the molecular and electronic structures (c.f., Fig. $2 a$, and $2 b$, respectively) and properties of the liquid crystal constituent molecules play the central role. On the other hand, for non-resonant nonlinearities associated with thermal-, density- and orientational-effects, a physical model in which liquid crystal molecules are represented as rigid rods and a host of physical parameters (temperature, density, and an order-parameter describing the intermolecular correlation effect, are usually adequate $[1,2]$.

A detailed formalism for describing second and third order electronic molec- 

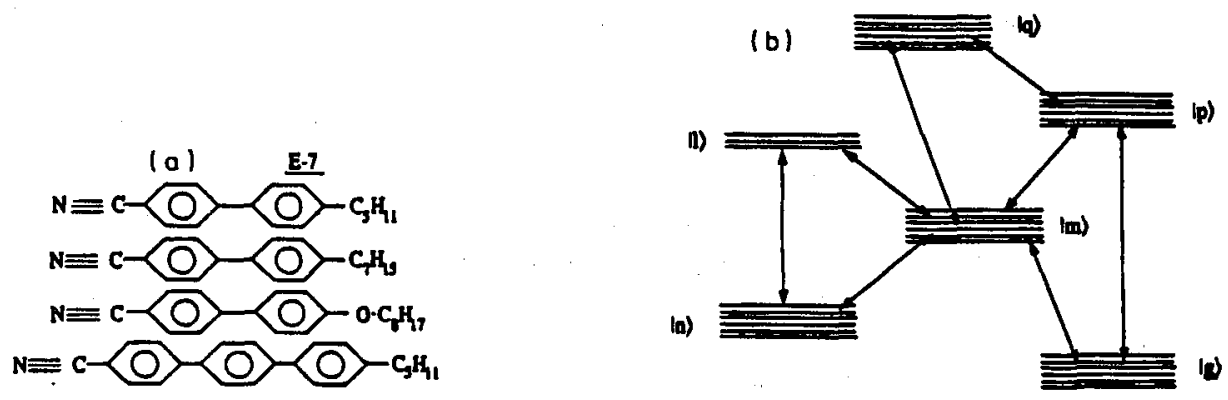

Fig. 2. (a) Molecular structure of room temperature nematic liquid crystal E7, a mixture of four liquid crystals in the nematic phase. (b) Multilever electronic transitions involved in the calculation of the second and third order nonlinear polarizabilities.

ular polarizabilities and macroscopic susceptibilities is given in Refs. [1, 2]. The exact expressions, of course, vary as the constituent molecules making up the liquid crystals, but they are generally of the form given below:

2nd order polarizability

$$
\begin{gathered}
\beta_{i j k}\left(-\left(\omega^{\prime}=\omega\right) ; \omega^{\prime}, \omega\right)=\sum_{n, m, l}\left(\frac{\rho_{m m}^{(0)}-\rho_{l l}^{(0)}}{\hbar}\right) \frac{d_{m n}^{i} d_{n l}^{i} d_{l m}^{k}}{\left[\Omega_{n m}-\left(\omega^{\prime}+\omega\right)\right]\left(\Omega_{l m}-\omega\right)} \\
-\sum_{n, m, l}\left(\frac{\rho_{l l}^{(0)}-\rho_{n n}^{(0)}}{\hbar}\right) \frac{d_{m n}^{i} d_{l m}^{j} d_{n l}^{k}}{\left[\Omega_{n m}-\left(\omega^{\prime}+\omega\right)\right]\left(\omega_{n l}-\omega\right)} ;
\end{gathered}
$$

2nd order susceptibility

$$
\chi_{i j k}^{(2)} \sim N\left(\bar{\alpha}_{i j k}^{(1)}\left\langle P_{1}\right\rangle+\bar{\alpha}_{i j k}^{(3)}\left\langle P_{3}\right\rangle\right)
$$

3rd order polarizability:

$$
\begin{aligned}
& \gamma_{i j k h}\left(-\left(\omega^{\prime \prime}+\omega^{\prime}+\omega\right) ; \omega^{\prime \prime} \omega^{\prime}, \omega\right)=\sum_{n m p q} \frac{\hbar^{-3} d_{m n}^{i}}{\omega_{n m}-\left(\omega^{\prime \prime}+\omega^{\prime}+\omega\right)} \\
& \times\left\{\frac{\left(\rho_{m m}^{(0)}-\rho_{q q}^{(0)}\right) d_{n p}^{j} d_{p q}^{k} d_{q m}^{h}}{\left(\omega_{p m}-\left(\omega^{\prime}+\omega\right)\right)\left(\omega_{q m}-\omega\right)}-\frac{\left(\rho_{q q}^{(0)}-\rho_{p p}^{(0)}\right) d_{n \dot{p}}^{j} d_{q m}^{k} d_{p q}^{h}}{\left(\omega_{p m}-\left(\omega^{\prime}+\omega\right)\right)\left(\omega_{p q}-\omega\right)}\right. \\
& \left.+\frac{\left(\rho_{p p}^{(0)}-\rho_{q q}^{(0)}\right) d_{p m}^{j} d_{n q}^{k} d_{q p}^{h}}{\left(\omega_{n p}-\left(\omega^{\prime}+\omega\right)\right)\left(\omega_{g p}-\omega\right)}+\frac{\left(\rho_{q q}^{(0)}-\rho_{n n}^{(0)}\right) d_{p m}^{j} d_{q p}^{k} d_{n q}^{h}}{\left(\omega_{n p}-\left(\omega^{\prime}+\omega\right)\right)\left(\omega_{n q}-\omega\right)}\right\}
\end{aligned}
$$

3rd order susceptibility (an example): 


$$
\chi_{i i i i}^{(3)} \sim N \bar{\alpha}_{i i i i}\left(\frac{1}{5}+\frac{4}{7}\left\langle P_{2}\right\rangle+\frac{8}{35}\left\langle P_{4}\right\rangle\right)
$$

where $\bar{\alpha}_{i j k}$ and $\bar{\alpha}_{i i i i}$ are the local field corrected linear combinations of the second and third order polarizabilities, $\beta_{i j k}$ and $\gamma_{i j k h}$, respectively. The molecular correlation effects, i.e., order, present in the liquid-crystal phases are reflected in the presence of $\left\langle P_{2}\right\rangle$ and $\left\langle P_{4}\right\rangle ; d_{m n}^{i}$ are the dipole matrix element connecting the $i$-th and the $j$-th level, $\omega$ is the incident laser frequency, $\omega_{i j}$ - the frequency of the $i-j$ transition, $\rho_{n n}^{(0)}, \rho_{l l}^{(0)}$, and $\rho_{m m}^{(0)}$ are the population density of the $n$-th, $l$-th and $m$-th state, respectively.

Besides these hyperpolarizabilities type of electronic nonlinearities, liquid crystals have also been shown to exhibit the Raman and Brillouin nonlinearities, c.f., Fig. 3. Stimulated Raman and Brillouin scatterings have been observed in a
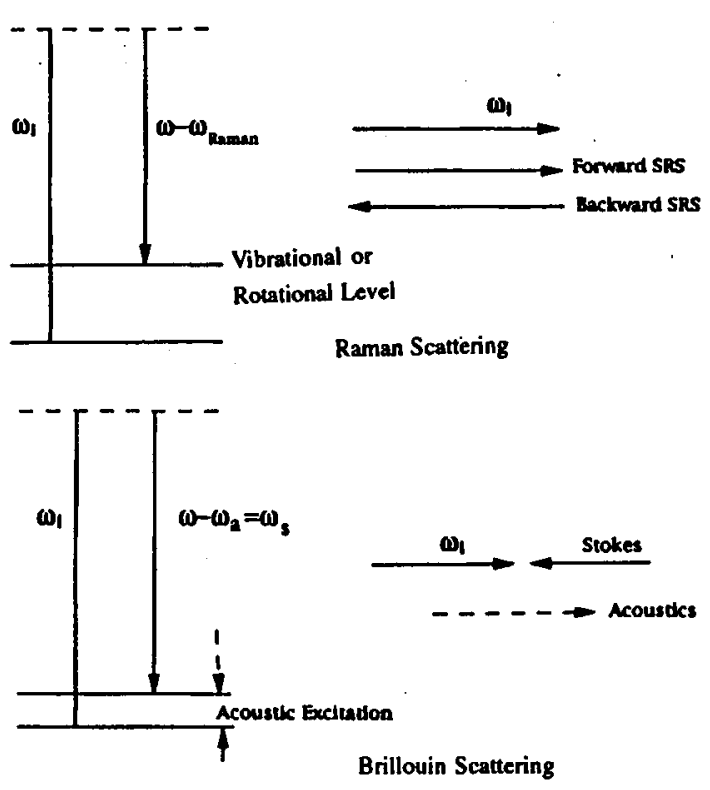

Fig. 3. Raman and Brillouin scattering process.

variety of liquid crystals in their ordered or isotropic phases in bulk form $[3,4]$. These previous studies have been performed basically for understanding nonlinear optical phenomena. On the other hand, the observations of stimulated Raman and Brillouin scattering in liquid crystalline fibers to be described in Ref. [2b] appear to have great application potentials.

The principal non-resonant nonlinearities arise from laser-induced molecular reorientation $(\Delta \theta)$, thermal $(\Delta T)$ - and density-charges $(\Delta \rho)$, all of which result in an (optical) intensity dependent index change $\Delta n$. These effects are summarized by the following equations [2]: 
1) orientational:

\section{Nematic phase}

$$
\begin{gathered}
\frac{\mu \partial^{2} \Delta \theta}{\partial t^{2}}+\gamma \frac{\partial \Delta \theta}{\partial t}+\frac{1}{2}\left(\gamma_{1}-\gamma_{2} \sin 2 \Delta \theta\right) \frac{\partial V}{\partial z} \\
=K \frac{\partial^{2} \Delta \theta}{\partial z^{2}}+\frac{\Delta \varepsilon E_{\mathrm{op}}^{2}}{8 \pi} \sin 2(\beta+\Delta \theta) ;
\end{gathered}
$$

Intensity dependent refractive index change:

$$
\Delta n=\frac{\mathrm{d} n}{\mathrm{~d} \theta} \Delta \theta
$$

Isotropic phase

$$
\gamma \frac{\partial Q_{\alpha \beta}}{\partial t}+A Q_{\alpha \beta}=\Delta \chi\left(3 E_{\alpha}^{*} E_{\beta}-|E|^{2} \delta_{\alpha \beta}\right) / 9 ;
$$

Intensity dependent refractive index change:

$$
\Delta n \approx \Delta \chi Q
$$

2) thermal:

$$
\rho_{0} c_{p} \frac{\partial(\Delta T)}{\partial t}-\gamma_{T} \nabla^{2}(\Delta T)-\frac{1}{B}\left(c_{p}-c_{v}\right) \frac{\partial(\Delta) \varrho}{\partial t}=\frac{\alpha n c}{4 \pi} r e\left(\boldsymbol{E}_{\mathrm{op}} \cdot \boldsymbol{E}_{\mathrm{op}}^{*}\right) ;
$$

Intensity dependent refractive index changes:

Nematic phase

$$
\Delta n=\frac{\mathrm{d} n}{\mathrm{~d} S} \frac{\mathrm{d} S}{\mathrm{~d} T} \Delta T+\frac{\mathrm{d} n}{\mathrm{~d} \rho} \frac{\mathrm{d} \rho}{\mathrm{d} T} \Delta T ;
$$

isotropic phase

$$
\Delta n=\frac{\mathrm{d} n}{\mathrm{~d} \rho} \frac{\mathrm{d} \rho}{\mathrm{d} T} \Delta T
$$

3) Density:

$$
\begin{gathered}
\frac{\partial^{2} \Delta \rho}{\partial t^{2}}+\frac{V_{s}^{2}}{\gamma} \nabla^{2}(\Delta \rho)+\frac{V_{s}^{2} B \rho_{0}}{\gamma} \nabla^{2}(\Delta T) \\
+\frac{\eta}{\rho_{0}} \frac{\partial \nabla^{2}(\Delta \rho)}{\partial t}=\frac{\gamma^{\mathrm{e}}}{8 \pi} \nabla^{2} E_{\mathrm{op}}^{2} ;
\end{gathered}
$$

Intensity dependent refractive index change:

$$
\Delta n=\frac{\mathrm{d} n}{\mathrm{~d} \rho} \Delta \rho .
$$

In Eqs. (5)-(8), $\mu$ is the molecule's moment of inertia, $v$ - the flow velocity, $K$ is the appropriate elastic constant, $\Delta \varepsilon-$ the dielectric anisotropy, $\beta$ is the angle between the optical field and the nematic liquid crystal director axis, $\gamma$ is the viscosity coefficient, $Q_{\alpha \beta}$ - the tensorial order parameter (for isotropic phase), $E_{\text {op }}$ - the optical electric field, $T_{\mathrm{c}}$ - the nematic $\rightarrow$ isotropic phase transition temperature, $S$ - the order parameter (for liquid-crystal phase), $\gamma_{\mathrm{T}}$ - the thermal conductivity, $\alpha-$ the absorption constant, $\rho_{0}-$ the density, $c_{p}-$ the specific heat, $B$ - the bulk modulus, $v_{\mathrm{s}}$ - the velocity of sound, $\gamma^{\mathrm{e}}-$ the electrostrictive coefficient. 
TABLE

\begin{tabular}{l|c|c|c}
\hline \hline $\begin{array}{l}\text { Nonlinear mechanism } \\
\text { (for both isotropic } \\
\text { and nematic phases } \\
\text { unless specified) }\end{array}$ & $\begin{array}{c}\text { Response times } \\
\text { (quoted here } \\
\text { characteristic } \\
\text { relaxation time) }\end{array}$ & $\begin{array}{c}\text { Nonlinearity } \\
\eta_{\text {laser }}>\tau_{\text {response }} \\
\chi^{(3)} \text { in esu unit }\end{array}$ & $\begin{array}{c}\text { Nonlinearity } \\
\eta_{\text {aser }}<\tau_{\text {response }} \\
\chi^{(3)} \text { in esu unit }\end{array}$ \\
\hline $\begin{array}{l}\text { Individual molecular } \\
\text { reorientation }\end{array}$ & $\tau_{\text {off }} \sim \mathrm{ps}$ & $\leq 10^{-12}$ & Same \\
\hline $\begin{array}{l}\text { Electronic hyper- } \\
\text { polarizability (see } \\
\text { section 3.8) }\end{array}$ & $\tau_{\text {off }}<\mathrm{ps}$ & $\begin{array}{c}\geq 10^{-12} \\
(\text { may be as } \\
\text { large as } 10^{-9}\end{array}$ & Same \\
\hline $\begin{array}{l}\text { Collective molecular } \\
\text { reorientation }\end{array}$ & & & \\
\hline - Nematic & $\tau_{\text {off }} \sim \mathrm{ms}$ to s & $\geq 10^{-4}$ & See Ref. [2] \\
\hline - Dye doped nematics & $\tau_{\text {off }} \sim \mathrm{ms}$ to s & $\geq 10^{-2}$ & See Ref. [5] \\
\hline - Isotropic & $\tau_{\text {off } \leq 10^{2} \mathrm{~ns}}$ & $\geq 10^{-9}$ & See Ref. [2] \\
\hline Density effect & $\tau_{\text {off } \leq 10^{2} \mathrm{~ns}}$ & $>10^{-7}$ & See Ref. [2] \\
\hline Thermal effect & & & \\
\hline - Nematic & $\tau_{\text {off } \geq 10^{2} \mu \mathrm{s}}$ & $\geq 10^{-5}$ & \\
\hline - Isotropic & $\tau_{\text {off } \leq 10^{2} \mu \mathrm{s}}$ & $\geq 10^{-7}$ & See Ref. [2] \\
\hline
\end{tabular}

Comment: All values quoted here are critically dependent on laser pulse duration, absorption, characteristic diffusion length, geomçtry, etc., as explained in the text, and may vary by one or two orders of magnitude for different liquid crystals or operating conditions.

Table summarizes these optical nonlinearities, their magnitudes and relaxation times scales. Also included in Table is the extraordinary large optical nonlinearity we recently observed in excited dye-molecules doped liquid irystals; nonlinearity characterized by a $\chi^{(3)}$ on the order of $\geq 10^{-2}$ esu of such system has been measured [5].

Furthermore, studies [6] have also shown that in conjuction with some surface aligining polymer film typically used in fabricating nematic liquid crystal sample, the laser induced liquid crystal reorientations can become permanent (and erasable by optical or other means).

\section{Nonlinear optical phenomena observed in liquid crystals}

Owing to the various mechanisms for optical nonlinearities present in the ordered as well as isotropic phases of liquid crystals, almost all nonlinear optical phenomena have been observed. Some of these phenomena were studied for their 
novelty, others have been developed into diagnostic tools or practical devices. In a somewhat arbitrary manner, these observed effects may be grouped together under the following general headings.

a) Self-focusing, -defocusing and -phase modulation,

b) optical wave mixing including harmonic generation, beam amplification and phase conjugation,

c) optical bistability, instability, switching and limiting,

e) nonlinear wa veguiding,

f) stimulated scattering.

In view of the rapid advances made by several research groups, it is likely that the following summary will be outdated shortly.

According, we limit our attention here to list only exemplary studies which are fundamentally interesting and/or practically important. The readers are referred to the literature quoted in the two major review articles $[1 ; 2]$ and $[7]$.

a) Self-focusing, -defocusing and -phase modulation Nematic phase

A quantitative study of the role played by the laser wavefront curvature and other geometrical/optical parameters on the transmitted far-field intensity profile $[8,9]$; demonstration of self-defocusing effect for optical limiting application [10]. Isotropic phase

Study of the interplay between liquid crystal reorientation time scales and transient self-focusing effects [11].

b) Optical wave mixings

(i) Degenerate optical wave mixings

Isotropic phase

Use of molecular reorientational nonlinearity in optical phase conjugation [12]; study of the influence of molecular structure on molecular reorientational nonlinearity [13].

Nematic phase

Optical phase conjugation [14]; optical beam amplifications involving orientational and thermal nonlinearities [15-17]; polarization switching and beam amplification employing the intensity dependent birefringence of liquid crystals [18].

(ii) Non-degenerate optical wave mixing

Isotropic phase

Theory and experimental demonstration of optical third-harmonic generation in cholesteric liquid crystal [19].

Nematic phase

Second harmonic generation with [20] or without dc field [21].

c) Optical bistability and switching

\section{Nematic}

Cavityless bistability employing self-phase modulation $[8,9]$; nonlinear Fabry-Perot bistability effect [22]; intrinsic optical bistability [23]; nonlinear optical switching near the total-internal reflection state [24, 25] bistability in guided wave geometry [26]. 
Besides these nonlinear optical phenomena, other noteworthy studies include: multiphoton absorption [27], stimulated scatterings [28], nonlinear fiber-fiber coupling [29], acousto-optic interference effect in dynamic grating difractions [30]; degenerate optical wave mixing in polymer dispersed liquid crystal film [31].

The references as well as the phenomena quoted above are by no means exhaustive; the reader, therefore, should consult the rcferences quoted therein for more literature.

In the following section, we have selected the topic of self-starting optical phase conjugation for discussion as it is both fundamentally interesting and potentially useful for developing coherent optical device.

\section{Self-starting optical phase conjugation}

Self-pumped optical phase conjugation, in which a single incident laser beam gencrates its phase conjugated replica via some optical wave mixing effect in a nonlinear optical material, is a fundamentally interesting and practically useful process. Usually, the signal originates as some coherently scattered noise from the pump laser beam, e.g., owing to scatterers in a crystal, spontaneous Brilloin scattering, etc. This noise signal interacts with the pump beam, and grows into a strong coherent signal. This phenomenon is commonly observed in stimulated Brillouin scattering involving high-power pulsed laser [32,33], and in photorefractive materials with low-power $\mathrm{cw}$ lasers [34,35]. More recently, self-pumped phase conjugation effect has also been observed in resonant media, using frequency shifted signal and pump field [36], or degenerate four-wave mixing process [37].

The first observation, to our knowledge, of self-pumped phase conjugation in a Kerr-like medium, using a non-resonant type of nonlinearity-stimulated thermal scattcring in a nematic liquid crystal was reported by our group recently [38]. Figure 4 depicts the experimental setup; the insert shows the wave vector matching condition for the degenerate wave mixing process. In this process, the input beam transverses the medium twice, once as an incident field $\boldsymbol{E}_{1}$ (along $\boldsymbol{K}_{1}$ direction), and then, upon reflection from the optical system behind the sample, as a reflected field $\boldsymbol{E}_{2}$ (along $\boldsymbol{K}_{2}$ ) direction. $\boldsymbol{E}_{1}$ and $\boldsymbol{E}_{2}$ are not necessarily coherent with respect to each other.

The mechanism for generating a phase conjugated signal is as follows. As shown in the insert of Fig. 4, the incident beam generates a noise source field $\boldsymbol{E}_{3}$ (along $\boldsymbol{K}_{3}$ ) which is coherent with respect to $\boldsymbol{E}_{1}$. Accordingly, $\boldsymbol{E}_{1}$ and $\boldsymbol{E}_{3}$ can interfere with each other, and produce an index grating. For the planar aligned nematic liquid crystal film used in this experiment, where the director axis $\hat{n}$ is normal to the plane of the figure (i.e., parallel to $\boldsymbol{E}_{1}, \boldsymbol{E}_{2}, \boldsymbol{E}_{4}$ ), the index grating is associated with the temperature-dependent extraordinary refractive index $n_{\mathrm{e}}(T)$ $[1,2]$. Similarly, the reflected field $\boldsymbol{E}_{2}$ will interfere with its coherent noise $\boldsymbol{E}_{4}$ and produce an index grating.

In general, the scattered noise $\boldsymbol{E}_{3}$ and $\boldsymbol{E}_{4}$ from the two "input" beams $\boldsymbol{E}_{1}$ and $\boldsymbol{E}_{2}$ contains various temporal and spatial frequency components. However, for a given crossing angle between the incident and reflected field, only the scattered noise components $\left(\boldsymbol{K}_{3}\right.$ and $\boldsymbol{K}_{4}$ ) which obey the wave vector matching condition 


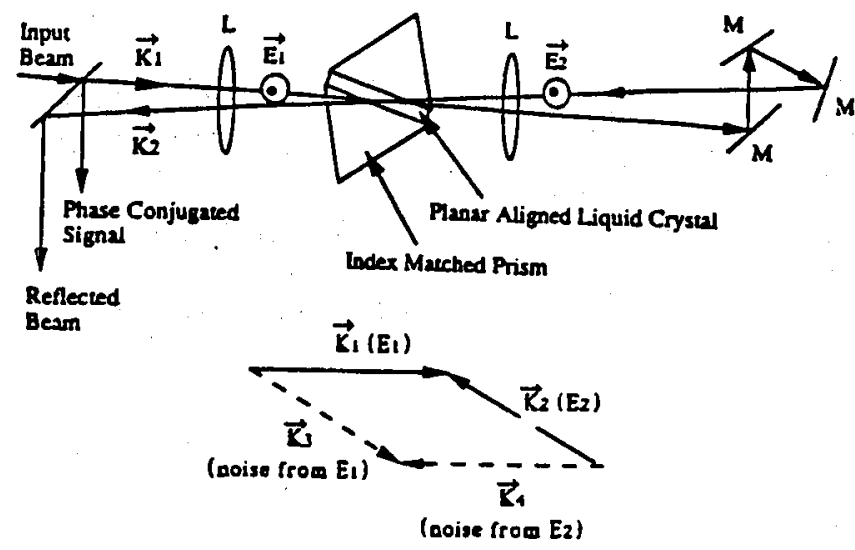

Fig. 4. Experimental setup for observing self-starting optical phase conjugation effect. Insert shows the wave-vector matching diagram.

as depicted in Fig. 4 will be to share a common grating and experience growth via the wave mixing effects.

This phase matched parametric process between two sets of waves $\left(\boldsymbol{E}_{1}, \boldsymbol{E}_{3}\right)$ and $\left(\boldsymbol{E}_{2}, \boldsymbol{E}_{4}\right)$ sharing a common thermal grating is similar to the ordinary-extraordinary wave mixing process analyzed in Ref. [39]. When the gains experienced by the noise fields exceed the loss, the process becomes a stimulated one with the resulting coherently generated field $\boldsymbol{E}_{4}$ comprising mainly of a phase conjugate reflection of $E_{1}$ (note: $K_{4}=-K_{1}$ ).

The liquid crystal used is a $150 \mu \mathrm{m}$ planar aligned dye-doped 5CB (PentylCyano-Biphenyl) cell enclosed by prisms where refractive index is 1.73 . The dye used is D16 (from EM Chemicals, NY) at a concentration of $0.5 \%$ by weight. The laser pulses is derived from an electronically chopped Argon laser $(1545 \AA)$, with a square-pulse duration of $2 \mathrm{~ms}$. The laser is linearly polarized, with the polarization vector parallel to the director-axis of the liquid crystal, i.e., it "sees" $n_{\mathrm{e}}-$ the extraordinary index. For $5 \mathrm{CB}, n_{\mathrm{e}} \approx 1.68$. The laser is obliquely incident on the cell, as shown in Fig. 4, so that the effective interaction length within the liquid crystal is about $1 \mathrm{~mm}$. As a result of the reflection loss at the glass liquid crystal interface, and scattering loss in the liquid crystal, only $5 \%$ of the incident beam is transmitted. The transmitted beam is re-collimated, and focused back into the cell, making an angle $v \approx 1^{\circ}$ with the incident beam. The spot diameter of the input laser at the liquid crystal is $0.5 \mathrm{~mm}$, whereas the reflected beam spot size is about five times smaller; at the overlap region, the intensities of the two beams are thus roughly equal. A beam splitter placed in the path of the incident and reflected beams is used to monitor simultaneously the phase-conjugated and the reflected signals.

A phase-conjugation reflection signal (which propagates along the reverse of the pump beam $\boldsymbol{E}_{1}$ ) becomes visible (c.f., Fig. 5) when the input laser power is about $400 \mathrm{~mW}$, and the cell temperature is within $5^{\circ}$ of $T_{\mathrm{c}}$, the phase transition temperature. Typically, the phase conjugation reflectivity is about $2 \%$ at an input 

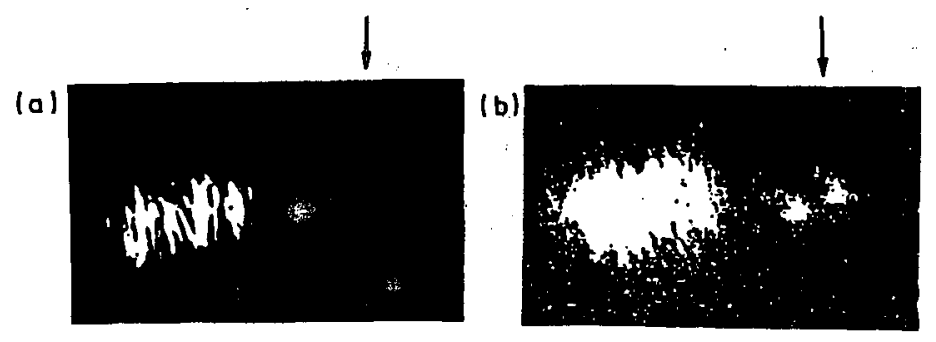

Fig. 5. Photograph of the reflected beam and phase conjugated signal. (a) At low power input, there is no phase conjugated signal (arrow indicates location). (b) At higher input power, phase conjugated signal appears. Double reflections are due to the glass-slide beam splitter used.

laser power of $800 \mathrm{~mW}$. It is noticed that in spite of the aberralions imparted by the input lens and other optics, which lead to strong aberration and large divergence on the incident and reflected bearns, the phase conjugated signal is spatially of the same quality as the input beam, and has about the same divergence. Such aberration correction property of optical phase conjugation makes the process very attractive for optical image or signal processing device application.

The main advantages offered by liquid crystals compared to other materials (photorefractive crystals, for example) that also allow self-starting optical phase conjugation are their broadband applicability (from visible to infrared) and relatively fast onset times at moderate optical power. More importantly, liquid crystals are also very low cost materials, compared to photorefractive crystals. Since the time of the writing of this article, we have also succeeded in using the stimulated orientational scattering effects [2b], instead of the thermal effects mentioned above, to realize similar self-starting phase conjugation effects. Details of these recent observations will be reported elsewhere.

This work is supported by grants from the National Science Foundation and the Air Force Philips Laboratory.

\section{References}

[1] I.C. Khoo, in: Progress in Optics, Vol. XXVI, Ed. E. Wolf, North-Holland, Amsterdam 1988, p. 107.

[2] (a) I.C. Khoo, S.T. Wu, Optics and Nonlinear Optics of Liquid Crystals, World Scientific, New Jersey 1993. See also (b) I.C. Khoo, Liquid Crystals: Physical Properties and Nonlinear Optical Phenomena, Wiley, New York 1994, in press.

[3] See, for example, G.K.L. Wong, Y.R. Shen, Phys Rev. Lett. 32, 527 (1974); see also D.N. Ghosh Roy, D.V.G.L.N. Rao, J. Appl. Phys. 59, 332 (1986).

[4] I.C. Khoo, R.R. Michael, P.Y. Yan, IEEE J. Quant. Electron. QE-23, 1344 (1987).

[5] I.C. Khoo, H. Li, Y. Liang, IEEE J. Quantum Electron. QE-29, 1444 (1993).

[6] W.M. Gibbons, P.J. Shannon, Shao-Tang Sun, B.J. Swetlin, Nature 351, 49 (1991); A.G.-S. Chen, D.J. Brady, Opt. Lett. 17, 1231 (1991); see also K. Ichimura, Y. Suzuki, T. Seki, Y. Kawanishi, T. Tamaki, K. Aoki, Jpn. J. Appl. Phys. 28, 289 (1989). 
[7] N.V. Tabiryan, A.V. Sukhov, B.Ya. Zeldovich, Mol. Cryst. Liq. Cryst. 136, 1 (1986).

[8] I.C. Khoo, J.Y. Hou, T.H. Liu, P.Y. Yan, R.R. Michael, G.M. Finn, J. Opt. Soc. Am. B 4, 886 (1987).

[9] I.C. Khoo, P.Y. Yan, T.II. Liu, S. Shepard, J.Y. Hou, Phys. Rev. A 29, 2756 (1984).

[10] I.C. Khoo, R.R. Michael, G.M. Finn, Appl. Phys. Lell. 52; 2108 (1988); I.C. Khoo, Sukho Lee, P. LoPresti, R.G. Lindquist, Hong Li, Int. J. Nonlinear Opt. Phys. 2, 559 (1993).

[11] G.K.L. Wong, Y.R. Shen, Phys. Rev. Lett. 32, 527 (1974).

[12] D. Fekete, J. Au Yeung, A. Yariv, Opt. Lett. 5, 51 (1980).

[13] P.A. Madden, F.C. Saunders, A.M. Scott, IEEE J. Quantum Electron. QE-22, 1287 (1986).

[14] I.C. Khoo, S.L. Zhuang, IEEE J. Quantum Electron. QE-18, 246 (1981); F. Sanchez, P.H. Kayoun, J.P. Huignard, J. Appl. Phys. 64, 26 (1988); see also L. Richard, J. Maurin, J.P. Huignard, Opt. Commun. 57, 365 (1986).

[15] I.C. Khoo, T.H. Liu, Phys. Rev. A 39, 4036 (1989).

[16] T.II. Liu, I.C. Khoo, IEEE J. Quantum Electron. QE-23, 171 (1987).

[17] I.C. Khoo, P.Y. Yan, G.M. Finn, T.H. Liu, R.R. Michael, J. Opt. Soc. Am B 5, 202 (1988).

[18] I.C. Khoo, Phys. Rev. Lett. 64, 2273 (1990).

[19] J.W. Shelton, Y.R. Shen, Phys. Rev. Lett. 25, 23 (1970); see also Phys. Rev. A 5, 1867 (1972) by the same authors.

[20] S.K. Saha, G.K. Wong, Opt. Commun. 30, 119 (1979).

[21] A.V. Sukhov, R.V. Timashev, JETP Lett. 51, 415 (1990); see also N.B. Baranova, B.Ya. Zcldovich, Dokl. Akad Nauk SSSR 263, 325 (1982) [Sov. Phys. Dokl. 27, 222 (1982)].

[22] I.C. Khoo, J.Y. Hou, R. Normandin, V.C.Y. So, Phys. Rev. A 27, 3251 (1983).

[23] A.J. Karn, S.M. Arakelian, Y.R. Shen, II.L. Ong, Phys. Rev. Lett. 57, 448 (1986).

[24] I.C. Khoo, Ping Zhou, R.R. Michael, R.G. Lindquist, R.J. Mansfield, IEEE J. Quantum Electron. QE-25, 1755 (1989).

[25] I.C. Khoo, W. Wang, F. Simoni, G. Cipparrone, D. Duca, J. Opt. Soc. Am. B 8, 1464 (1991).

[26] II. Vach, C.T. Seaton, G.I. Stegeman, I.C. Khoo, Opt. Lett. 9, 238 (1984). See also E.S. Goldburt, P.St.J. Russell, Appl. Phys. Lell. 46, 338 (1985).

[27] M.J. Soileau, S. Guha, W.E. Williams, E.W. Van Stryland, II. Vanherzeele, Mol. Cryst. Liq. Cryst. 127, 321 (1985); H.J. Eichler, R. MacDonald, B. Trösken, Mol. Cryst. Liq. Cryst. 231, 1 (1993).

[28] D.N.G. Roy, D.V.G.L.N. Rao, J. Appl. Phys. 59, 332 (1986); I.C. Khoo, R.R. Michael, P.Y. Yan, IEEE J. Quantum Electron. QE-23, 1344 (1987); see also A.V. Sukhov, Mol. Cryst. Liq. Cryst. 185, 227 (1990); see also Ref. [1] which contains many references on stimulated scallerings.

[29] I.C. Khoo, R. Normandin, J. Appl. Phys. 65, 2566 (1989); see also Z.K. Ioannidis, I.P. Giles, C. Bowry, Appl. Opt. 30, 328 (1991).

[30] I.C. Khoo, R.G. Lindquist, R.R. Michael, R.J. Mansfield, P. LoPresti, J. Appl. Phys. 69, 3853 (1991); see also work by I.C. Khoo, R. Normandin quoted in Ref. [1]. 
[31] F. Simoni, G. Cipparone, D. Duca, I.C. Khoo, Opt. Lelt. 16, 360 (1991).

[32] B.Ya. Zeldovich, N.F. Pilipetsky, V.V. Shkunov, Principles of Phase Conjugation, Springer Series in Optical Sciences, Vol. 42, Springer Verlag, Berlin 1985.

[33] K.D. Ridley, A.M. Scott, Opt. Commun. 76, 406 (1990); Int. J. Nonlinear Opt. Phys. 1, 563 (1992); see also R.W. Boyd, Nonlinear Optics, Academic Press, New York 1992.

[34] J. Feinberg, Opt. Lelt. 7, 486 (1982).

[35] J.O. White, M. Cronin-Golomb, B. Fischer, A. Yariv, Appl. Phys. Lett. 40, 450 (1982); M. Cronin-Golomb, B. Fisher, J.O. White, A. Yariv, IEEE J. Quantum Electron. QE-20, 12 (1984); Appl. Phys. Lett. 42, 919 (1983).

[36] C.J. Gaeta, J.F. Lam; R.C. Lind, Opt. Lett. 14, 245 (1989).

[37] M. Vallet, M. Pinard, G. Grynberg, Opt. Lett. 16, 1071 (1991).

[38] I.C. Khoo, Hong Li, Yu Liang, Opt. Lett. 18, 3 (1993).

[39] I.C. Khoo, Phys. Rev. Lett. 64, 2273 (1990); see also B.Ya. Zel'dovich, N.V. Tabiryan, JETP Lett. 30, 478 (1979). 